Proceedings of SALT 20: 525-543, 2010

\title{
Quantity implicature and access to scalar alternatives in language acquisition*
}

\author{
David Barner \\ University of California, San Diego
}

\author{
Neon Brooks \\ University of Chicago
}

\author{
Alan Bale \\ Concordia University
}

\begin{abstract}
When faced with a sentence like Some of the toys are on the table, adults, but not preschoolers, compute a scalar implicature, taking the sentence to imply that not all the toys are on the table. This paper explores the hypothesis that children fail to compute scalar implicatures because they lack knowledge of the relevant scalar alternatives to words like some. Four-year-olds were shown pictures in which three out of three objects fit a description (e.g., three animals reading), and were asked to evaluate statements that relied on context-independent alternatives (e.g., knowing that all is an alternative to some for the utterance Some of the animals are reading) or contextual alternatives (e.g., knowing that the set of all three visible animals is an alternative to a set of two for the utterance Only the cat and the dog are reading). Children failed to reject the false statements containing context-independent scales even when the word only was used (e.g., only some), but correctly rejected equivalent statements containing contextual alternatives (e.g., only the cat and the dog). These results support the hypothesis that children's difficulties with scalar implicature are due to a failure to generate relevant alternatives for specific scales. Consequences for number word learning are also discussed.
\end{abstract}

Keywords: inference, scalar implicature, word learning, counting, exactness, language acquisition, semantic development, pragmatic development

\section{Introduction}

When presented with sentences containing scalar items like some, 5-year-old children, unlike adults, have trouble strengthening their meanings (e.g., strengthening some to some but not all). This difficulty has often been attributed to an underdeveloped processing ability or pragmatic competence (see Chierchia, Crain, Guasti,

* We would like to thank Asaf Bachrach, Charles Reiss, Jennifer Audet, and two anonymous reviewers for their help and useful comments on previous drafts of this paper. We would also like to thank the organizers and participants at SALT 2010.

C2010 D. Barner, N. Brooks \& A. Bale 
Gualmini \& Meroni 2001; Musolino 2006; Musolino \& Lidz 2006; Papafragou 2006; Papafragou \& Musolino 2003; Papafragou \& Tantalou 2004; Pouscoulous, Noveck, Politzer \& Bastide 2007). This paper explores an alternative hypothesis that children fail to compute scalar implicatures because they lack knowledge of the relevant scalar alternatives to words like some (see Barner \& Bachrach 2010). At the heart of this conjecture is the thesis that knowledge of scales goes beyond knowledge of lexical meanings and a capacity to compare sentences on the basis of informativeness. In addition to this, children must learn which lexical items are members of scales, and thus serve as relevant alternatives when interpreting sentences.

Section 3 outlines an experiment which tested our hypothesis. In the experiment, four-year-olds were shown pictures in which three out of three objects fit a description (e.g., three animals reading), and were asked to evaluate statements that relied on context-independent alternatives (e.g. only some of the animals are reading) or contextual alternatives (e.g. only the cat and the dog are reading). The word only was used to grammatically force a strengthened meaning. As described in section 4, children failed to reject the false statements containing context-independent scales even when the word only was used (e.g., only some), but correctly rejected the statements containing contextual alternatives when strengthened by only (e.g., only the cat and the dog). These results support the hypothesis that children's difficulties with scalar implicature are not related to processing constraints or other pragmatic limitations, but instead are due to a failure to generate relevant alternatives for specific scales. These results have interesting consequences for number word learning, as discussed in section 5 .

\section{Background}

A large number of studies starting in the early 1980's have found that children, unlike adults, often fail to derive scalar implicatures. This has been shown for many scalar contrasts, including might vs. must (Noveck 2001), a vs. some (Barner, Chow \& Yang 2009), some vs. all (Huang \& Snedeker 2009b,a; Hurewitz, Papafragou, Gleitman \& Gelman 2006; Musolino 2004; Noveck 2001; Papafragou \& Musolino 2003; Smith 1980), and or vs. and (Chierchia et al. 2001; Gualmini, Crain, Meroni, Chierchia \& Guasti 2001). For example, in a study by Papafragou \& Musolino (2003), 5-year-old children were shown a scene with three horses, in which all three animals successfully jumped over a log. When children were asked whether the sentence, Some of the horses jumped over the log was a good description of the event, most said yes. Adults, in contrast, denied that this was a good description, since all of the horses jumped over the log. Adults, unlike the 5-year-old children,

computed a scalar implicature. Calculations of scalar implicatures minimally involve 
Quantity implicature and access to scalar alternatives

the following four steps:

I. Compute basic meaning of a sentence $\mathbf{S}$ containing $\mathbf{L}$, a scalar item. (e.g., a meaning similar to At least some of the horses jumped over the log.)

II. Generate a set of alternatives $\left(a_{1}, a_{2}, \ldots, a_{n}\right)$ to $\mathbf{S}$, called Salt. These are all the sentences that can be generated by replacing $\mathbf{L}$ with its scalar alternatives. (e.g., Salt $=\{$ All of the horses jumped over the log, ., etc. $\}$ )

III. Restrict the alternatives in Salt by removing any alternative that is entailed by the original utterance $\mathbf{S}$. Call this restricted set $\mathbf{S}^{*}$.

IV. Strengthen the basic meaning of $\mathbf{S}$ (containing $\mathbf{L}$ ) with the negation of all of the members of $\mathbf{S}^{*}$. (e.g., Some but not all of the horses jumped over the log.)

Interestingly, children do not always fail to derive strengthened interpretations. Papafragou and Musolino found that children provided adult-like responses when tested with numerals. Children denied that Two of the horses jumped over the log when three horses did. Thus, although children failed to have adult-like responses with some and all, they interpret numerals with an exact-meaning just like adults.

What accounts for children's frequent failure to compute implicatures, and why do they fail with the quantifiers some and all, but not with numerals? Previous studies have suggested various factors that might affect children's derivation of implicatures, including limitations on working memory, limited understanding of context and meta-linguistic tasks, and the salience or availability of relevant scalar alternatives (see Chierchia et al. 2001; Musolino 2006; Musolino \& Lidz 2006; Papafragou 2006; Papafragou \& Musolino 2003; Papafragou \& Tantalou, 2002; Pouscoulous et al. 2007). According to Papafragou and Musolino, since each of these factors might limit children's computation of implicatures, and since children readily assign exact interpretations to numerals, children must not be using implicatures to derive exact meanings of numerals. Instead, by their view, the difference between quantifiers and numerals is due to the fact that numerals, unlike quantifiers, have lexically strengthened, exact, meanings (see also Huang, Snedeker \& Spelke under review; Hurewitz et al. 2006; see Breheny 2008, for an example of an exact semantics).

It is uncontroversial that context affects when and whether children (or adults, for that matter) will compute implicatures (e.g., Guasti, Chierchia, Crain, Foppolo, Gualmini \& Meroni 2005; Papafragou \& Musolino 2003; Musolino 2006). It is also known that working memory capacity grows over the course of development (e.g., Gathercle \& Baddeley 1990). Finally, it is known that adults take longer to compute implicatures than to arrive at an utterance's literal meaning, suggesting that additional processes are involved (Rips 1975; Noveck \& Posada 2003; Huang \& Snedeker 2009a,b). Nevertheless, the role of these factors in children's pragmatic 
difficulties has not been empirically established. First, although previous studies find that implicatures are more likely in some contexts than others, the fact that strong contextual cues can push children towards one interpretation over another does not show that their difficulties are due to contextual misunderstanding. Instead, strong contextual cues may compensate or mask difficulties that originate elsewhere in the process of deriving implicatures. ${ }^{1}$

Second, there is currently no direct evidence that working memory or processing constraints are responsible for children's difficulty computing implicatures. Studies that document additional processing time for implicatures do not suggest that such additional processing is taxing, nor that it is sufficient to cause difficulty for children. Instead, the claim is only that implicatures are not immediate (and therefore, by these accounts, not part of the literal semantics). Studies that attribute children's problems to processing limits (e.g., Chierchia et al. 2001; Reinhart 2004; Pouscoulous et al. 2007) do not actually assess working memory or processing capacity, and therefore do not test whether individual differences in capacity predict differences in pragmatic abilities. $^{2}$ Also, these studies do not provide a model of how processing limits, per se, could generate the effects found - i.e., which specific processes are affected and how.

For example, in their study of implicature and disjunction, Chierchia et al. (2001) tested 3- to 6-year-old children's interpretation of or, and whether they could distinguish its weak, inclusive, interpretation from its strong, exclusive one. Unlike adults, when children of this age were told Every boy chose a skateboard or a bike, they accepted situations in which each boy chose both objects. Thus, they accepted the weak inclusive interpretation of $o r$, when adults did not. However, when children were explicitly presented a sentence containing and as an alternative, they strongly preferred it over a sentence containing or. According to Chierchia et al. (2001), children's failure to compute implicatures under normal circumstances is "due to the processing cost involved in constructing alternative representations of the sentence they were presented with." (p. 167)

1 As shown by Musolino, children readily assign numerals a lower-bounded "at least" interpretation in the context of familiar games (in which the winner must get some minimum number of points). These studies ask children to judge whether a Troll is the winner. However, it is unknown whether these same children would agree that a Troll who scored 5 baskets also scored 3. Children may know how to determine a winner in such contexts without actually thinking that three can be assigned a weak interpretation of "at least three."

2 The evidence from Pouscoulous et al. 2007 for a processing cost comes from the observation that some tasks (i.e., creating a set) are more likely to elicit adult behaviour than others (i.e., truth value judgment), and that some lexical items are used in more complex syntactic constructions (like the partitive). However, the studies fail to test whether the tasks or words used actually differ in their demands on processing, or how such a processing limit would explain the findings (i.e., which processes were limited, and how). 
Quantity implicature and access to scalar alternatives

This study clearly shows that when children are presented with two alternative descriptions of a scene they prefer stronger, more informative descriptions. However, it does not provide evidence that processing limits, per se, are the source of children's difficulty computing implicatures. Instead, it shows that in some way, children's access to alternatives limits implicature, leaving open why access is limited. As we will argue, this result, and others in the literature, are consistent with the hypothesis that children lack knowledge of scales, and thus which lexical items are relevant alternatives in a given context (see Papafragou \& Tantalou 2004). By this view, children may know the meanings of scalar terms and their relative strength as descriptions, but lack knowledge of which items constitute scales, and therefore are unable to access relevant alternatives when processing sentences.

According to this hypothesis, knowledge of scales goes beyond knowledge of lexical meanings and a capacity to identify which of two descriptions is more informative. Children must learn arbitrary restrictions to scales, such that not all possible alternatives are considered when computing implicatures. This hypothesis is motivated not only be developmental data, but also by previous observations that restrictions to scales are necessary (Horn 1972, 1989; Hirschberg 1985; Katzir 2007; Matsumoto 1995). First, without restrictions to scales, language users might consider an unbounded number of stronger alternatives to a given utterance, resulting in a problem of computational complexity. Second, if arbitrary restrictions were not placed on scales, and only semantic criteria like informativeness were considered, then many spurious and unattested inferences would result. For example, although two apples is a stronger and more informative description than some apples in a context containing two apples, the words some and two are clearly not scale mates. If they were, then we would expect two to restrict and strengthen the interpretation of some - e.g., for the expression John ate some apples to rule out John ate two apples (and for that matter, any other alternative that contains a numeral, resulting in a situation where some can only mean exactly one). However, even young children avoid this error, and assign numerals to a unique scale even before they know their specific meanings. Although 2-year-olds use known numeral meanings to restrict their interpretation of unknown numerals, they do not use them to restrict known quantifiers like some (Condry \& Spelke 2008) or novel quantifiers like toma (Wynn 1992).

A failure to represent lexical items as members of psychological scales could explain numerous results in the literature, and also the apparent discrepancy between children's difficulty with implicatures and their relatively sophisticated use of pragmatic cues elsewhere in language acquisition. For example, this account, as noted by Barner \& Bachrach (2010), could explain children's ability to assign exact interpretations to numerals, which belong to an explicitly memorized list of scalar alternatives - the count list. As already noted, theories that view scales as emergent 
properties of semantic meaning are forced to assign numerals a qualitatively different analysis from other quantifiers, since children readily access exact meanings of numerals but cannot strengthen quantifiers used in equivalent contexts. However, by the view that scales are constructed by associating scale mates to one another, numerals and quantifiers can be assigned a uniform analysis, unlike any previous account.

In their study, Barner \& Bachrach (2010) argued that very young children beginning at 2 years of age - routinely make inferences that are similar in structure to scalar implicatures when interpreting unknown numerals (i.e., numerals for which they have not yet acquired an adult-like meaning). As noted earlier, when a child who knows the meaning of one is shown two sets - e.g., one containing one balloon, and the other containing five - they systematically point to the larger set when asked to find five balloons. However, they do not do so when asked to find blicket balloons. According to Wynn (1992), "Since all the children knew that the word 'one' refers to a single item, then if they knew that, for example, the word 'five' refers to a numerosity, they should infer that it does not refer to a single item since they already have a word for the numerosity one." (p. 229)

This inference - that five refers to the larger set by virtue of not referring to one - requires all of the processing resources that an ordinary implicature would require, as well as several of the same steps. The child must generate a weak meaning for five (Step I), generate one as an alternative (Step II), and strengthen the interpretation of five by negating one (Step IV). The only missing component of implicature is that, typically, weaker items are strengthened by appeal to stronger ones (something that is impossible when interpreting unknown numerals, since stronger words have not yet been acquired). Still, once children acquire a meaning for two, they should be in a position to compute an implicature for one, meaning that even 2-year-olds could compute implicatures to derive exact meanings for numerals (for evidence that this is, indeed, what children do, see Barner \& Bachrach 2010). As far as we can see, there is no difference in the processing demands required by these welldocumented inferences and the inferences required by scalar implicature. The only salient difference is the particular scale being processed - i.e., numerals rather than quantifiers.

Critically, numerous studies of early number word acquisition find that children begin to explicitly memorize a count list before they learn any numeral meanings, and recite it like the alphabet (Fuson 1988). In contrast, no child is taught to recite quantifiers in a song. Thus, when interpreting utterances containing some, children may be unaware that their knowledge of all is relevant, since some and all have not been associated as scalar alternatives, their semantic properties notwithstanding. For the same reason that children (or adults) do not treat two as an alternative to some, they also do not treat all as relevant. For them, these two expressions differ in 
Quantity implicature and access to scalar alternatives

informativeness, but are simply not relevant alternatives when interpreting sentences.

Currently, there is little evidence outside the cases of numerals and quantifiers to assess the role of scalar knowledge in the development of children's pragmatic abilities. The present study widens the scope of this debate by contrasting children's interpretation of some, whose scale members are specified in a context-independent way (i.e., all is always a scale member with some), with their interpretation of words that have contextually specified alternatives (for discussion, see Hirschberg 1985). Previous studies find that young children are able to "augment" or strengthen utterances that rely on accessing contextual alternatives. For example, Goro, Minai \& Crain $(2005,2006)$ found that English-speaking 3- to 6-year-olds correctly rejected sentences like Only Bunny Rabbit will eat a carrot or a pepper in contexts where another character chose a pepper. Crain and colleagues concluded that such children must be computing alternative contrast sets in order to strengthen these sentences. Further, they concluded that "children have adult-like knowledge about the semantics of only, and are able to compute its complex semantic interaction with the interpretation of or." (Crain, Goro \& Minai 2007) This is because, without the word only, such a sentence would be felicitous whether or not another character was eating a pepper, so long as the Bunny was. ${ }^{3}$

As Crain and others have noted, the semantics of only mirrors the algorithm for calculating scalar implicatures. This fact allows us to isolate the role that knowledge of relevant alternatives plays in implicature. As with implicatures, only triggers the negation of alternative sentences. For example, consider the sentences in (1b) and (1c).

(1) a. John: Did you eat my cake?

b. Mary: I ate some of it.

c. Mary: I ate only some of the cake.

Both sentences in (1b) and (1c) indicates that Mary did not eat all of the cake. The difference between the sentences is that in (1c) the denial of the alternative "I ate all of the cake" is logically entailed by the sentence's core, literal meaning (it is not merely implied). Still, in order for this entailment relation to be realized, the listener must generate all as a relevant alternative in order to negate it (as in the case of implicature). Thus, although only makes the negation of alternatives obligatory and semantic, rather than optional and pragmatic, it still requires that the relevant alternatives be generated before they are negated. Therefore, evidence that children

3 Crain and colleagues also note the difficulties children have with only when multiple dimensions of contrast are possible - e.g., only the cat is holding a flag (when a duck is holding a flag and a balloon). However, as they show in the Bunny example, such errors do not arise when only one dimension of contrast is salient. 
comprehend only but fail to strengthen sentences containing only some would suggest that their difficulty may be caused by a failure to access scalar alternatives, rather than a general inability to reason pragmatically.

In our study, we contrasted children's interpretation of sentences containing some to their interpretation of sentences containing contextually-specified alternatives. For example, we asked questions like those in (2), for a situation in which three animals (a dog, a cat, and a cow) are sleeping:

(2) a. Are some of the animals sleeping?

b. Are only some of the animals sleeping?

c. Are the dog and the cat sleeping?

d. Are only the dog and the cat sleeping?

If children's difficulty computing implicatures for context-independent scales like $\langle$ some, all $\rangle$ is due to a failure to generate relevant alternatives, then they should accept statements like (2a) and (2b) regardless of whether only is present. They should fail to construct the alternative sentence containing all, and therefore be unable to strengthen either sentence. In contrast, children should have no difficulty strengthening a sentence like $(2 \mathrm{~d})$, since the alternative contrast set is contextually specified. ${ }^{4}$ Evidence of this kind would suggest that knowledge of alternatives critically limits children's ability to interpret scalar items like some.

\section{Methods}

\subsection{Participants}

We recruited 60 4-year-old native speakers of English (27 girls; $M=4 ; 6$, range $=$ $4 ; 1-5 ; 0)$ by phone, through daycares, or in children's museums in the greater San Diego area. Two additional children were excluded due to failure to complete the task.

\subsection{Stimuli}

Stimuli were twelve pictures, each depicting a scene of three items. Four cards were used in a familiarization phase, and eight in the test phase. Familiarization cards

4 Typically sentences like (2c) are only strengthened if a preceding context is provided in which a presupposition exists that all three might be reading. For example, if asked, Are the cat, the dog, and the cow reading?, a response of, The cat and the dog are reading would imply that the cow is not. This confirms that contextual alternatives are created only when required contextually, either by preceding sentences or the use of a focus element like only. 
Quantity implicature and access to scalar alternatives
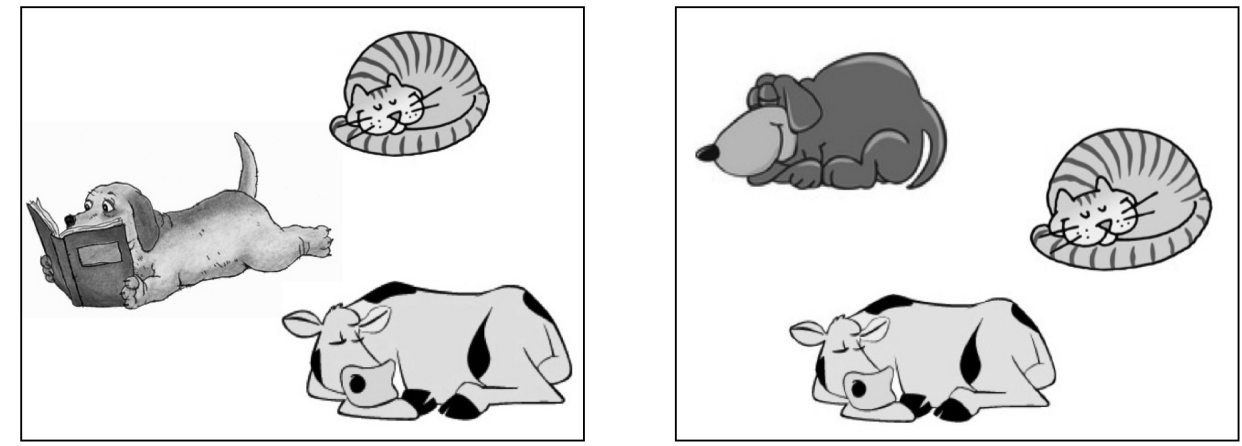

Figure 1 Examples of test stimuli in which two out of three, and three out of three, animals are sleeping.

depicted sets of animals with distinct characteristics, such as colour or clothing. The test cards depicted four scenes (in $\mathrm{a}-\mathrm{d}$ ).

a) Cookie Monster holding fruit (an orange, an apple, and a banana)

b) Animals sleeping (a dog, a cat, and a cow)

c) Animals reading (a dog, a cat, and a rabbit)

d) Toys on a table (a ball, a drum, and a train)

Two versions of each scene were created: one in which all three items shared a common property (e.g., all three animals are sleeping), and one in which two of the three items shared a common property (e.g., two animals are sleeping and one is reading). An example is provided in Figure 1.

\subsection{Procedure}

Children were seated with an experimenter at a child-sized table and were told that they would play a game with pictures. First, they were shown the four familiarization cards one at a time and asked to identify each animal on the card (What's this? That's right, it's a cow!). If the child labeled an animal incorrectly, they were told the correct response and encouraged to repeat it after the experimenter (That's a cow, can you say 'cow'?). Children were then asked a yes/no question, for which there was only one right answer (e.g., Is the cow wearing a hat? when the fish is wearing a hat). This familiarization exercise was designed to accustom children to answering 
both yes and no to questions. If a child answered any of these questions incorrectly, the experimenter moved on to the next familiarization card, but returned to the problematic card after completing the remaining familiarization trials. If a child failed twice on any single familiarization trial, the experimenter ended the testing session.

At test, children were given nine trials using the test cards, presented in one of two counterbalanced orders. As in the familiarization phase, children were first asked to identify all of the items in the picture and then to evaluate the truth-value of a statement.

Children were randomly assigned to one of four test conditions in a $2 \times 2$ betweensubjects design. The first factor, Alternative Type, determined whether children heard context-independent alternatives (i.e., some and $a l l$ ), or contextual alternatives (object labels, such as $d o g$ and cat) in the test questions. The second factor, Grammatical Strengthening, determined whether or not test questions contained the word only.

There were 9 questions in each condition. On "2-Item False" questions, children were shown a picture in which only two of the three items fit a description, and were asked whether the description was true for all the items (e.g., Are all of the animals sleeping? or Are the dog, the cat and the cow sleeping?). These were used as control trials, assuring that 'no' would be the correct answer on at least 1/3 of trials, and were presented without only in all conditions. On "2-Item True" questions, children saw pictures where two of the three items fit a description, and were asked whether the description was true for that subset of items (e.g., Are (only) some of the animals sleeping? or Are (only) the cat and the cow sleeping?). Lastly, on "3-Item Test" questions, children were shown pictures in which all three items fit the description, and asked whether the description was true for a subset of the items (e.g., Are (only) some of the animals sleeping? or Are (only) the cat and the cow sleeping?).

Neither the word only nor the quantifier was emphasized by the experimenter's prosody.

\section{Results}

The use of the word only had a significant effect on how children interpreted sentences involving contextual alternatives, but had no effect on their interpretation of sentences involving context-independent alternatives (some and all).

First, consider the data for Context-Independent alternatives (see Figure 2). In contexts where 2 of 3 items fit a description (e.g., two out of three animals are sleeping), children correctly agreed to sentences like, Are some of the animals sleeping? (2-Item True trials) on $80.0 \%$ of trials, and correctly denied that all of the animals were sleeping (2-Item False trials) on $87.2 \%$ of trials. On 2-Item True trials, use of the word only had no significant effect on children's judgments 
Quantity implicature and access to scalar alternatives

$(t(28)=0.00, p>0.05)$. Only was never used in 2-Item False trials, so it is not surprising that there was no difference between children's responses in the only and no-only conditions $(t(28)=0.96, p>0.05)$. Critically, on 3-Item Test trials, children in the Context-Independent Alternatives conditions behaved like children in previous studies of scalar implicature: when all three animals were sleeping and children were asked, Are some of the animals sleeping? they did not strengthen the utterance. Children in this condition accepted 3-Item Test trials on $66.6 \%$ of trials. Their responses did not differ significantly between 2-Item True trials and 3-Item Test trials $(t(14)=1.0, p>.3)$, suggesting that they were equally likely to agree that some animals were reading when all three of them were as when only two were. The insertion of only did not improve matters: there was no significant difference between strengthened and unstrengthened conditions on 3-Item Test trials $(t(28)=.16, p>.8)$. For example, when three animals were reading, children were equally likely to say yes when asked, Are only some of the animals sleeping? as when asked Are some of the animals sleeping?.

Next consider the data for Contextual Alternatives (Figure 3). As in the ContextIndependent condition, children answered control questions correctly: they agreed to 2-Item True trials $95.8 \%$ of the time, and correctly rejected 2-Item False trials $80.5 \%$ of the time. Use of the word only had no significant effect on children's judgments on 2-Item True trials $(t(28)=1.62, p>.05)$ nor on 2-Item False trials $(t(28)=0.57, p>0.05)$. In contrast, on the critical 3-Item Test trials, children tested with contextual alternatives were highly sensitive to the presence of only. Children almost always said yes when asked, Are the cat and the cow sleeping? (92.9\% of trials) but rarely said yes when only was added - Are only the cat and the cow sleeping? ( $14 \%$ of trials; $t(28)=8.98, p<.001)$. When only was added, there was also a significant difference between the number of yes responses in the 2-Item True and 3-Item Test conditions $(t(30)=9.27, p<0.001)$.

To compare results across conditions, data were entered into a linear logistic regression with Trial Type ("2-Item False" vs. "2-Item True" vs. "3-Item Test") as a within-subjects variable and Scale Type (Context-Independent vs. Contextual) and Grammatical Strengthening (only vs. no-only) as between-subjects variables. The model found significant main effects for all factors and all two-way interactions (all $p s<0.001)$. Critically, there was also a highly significant three-way interaction between Trial Type, Scale Type, and Grammatical Strengthening $(z=-3.29, p<$ .001). Children were significantly more likely to accept a critical 3-Item-test sentence if the sentence was grammatically strengthened and had contextual alternatives than if it was strengthened and was presented with context-independent alternatives. Thus, whereas only had a very large impact on children's interpretation of utterances including contextual alternatives, it had no effect at all when children interpreted 
D. Barner, N. Brooks \& A. Bale

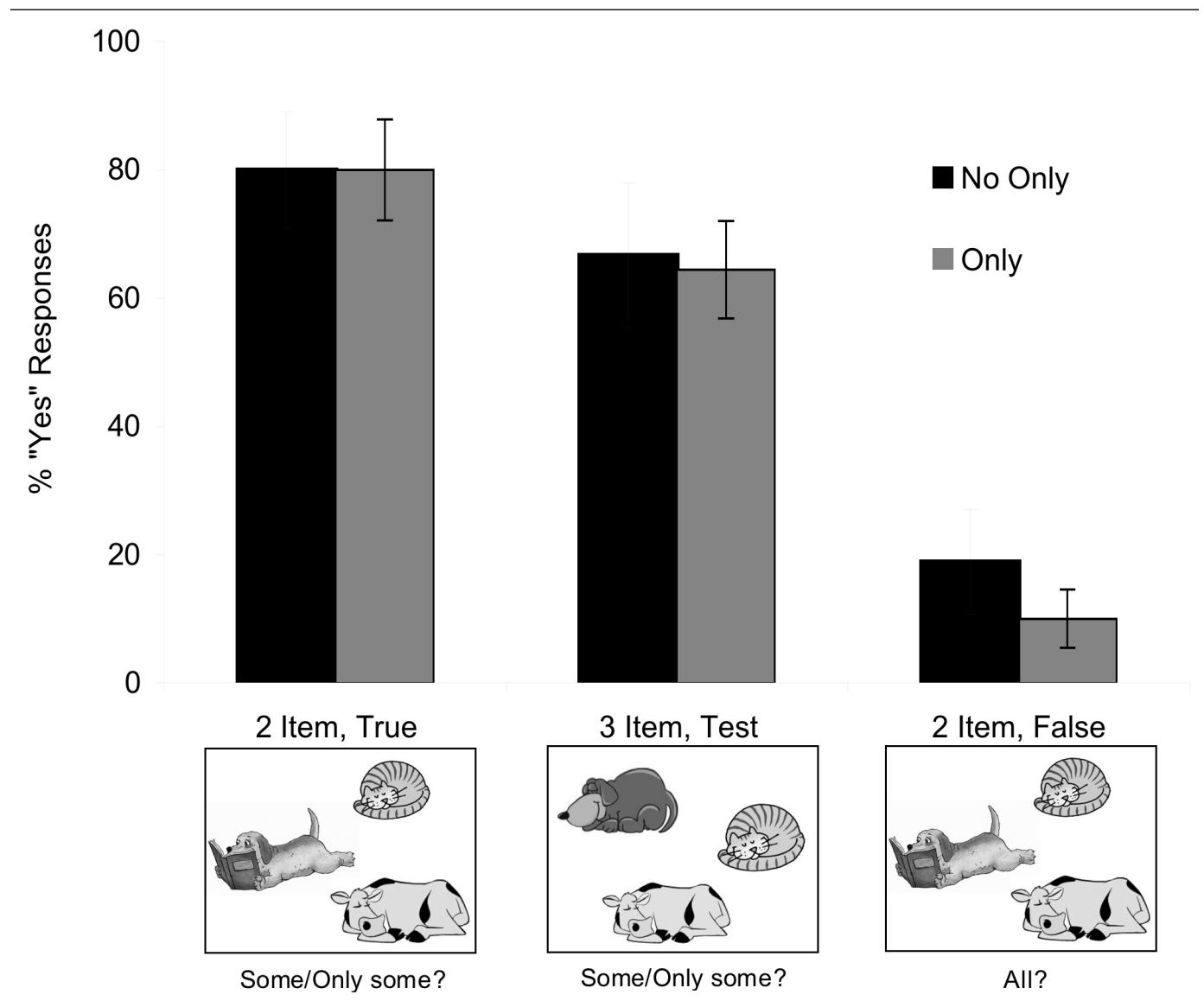

Figure 2 Percentage of children who said yes to questions in the ContextIndependent alternatives conditions. Error bars represent standard error. 
Quantity implicature and access to scalar alternatives

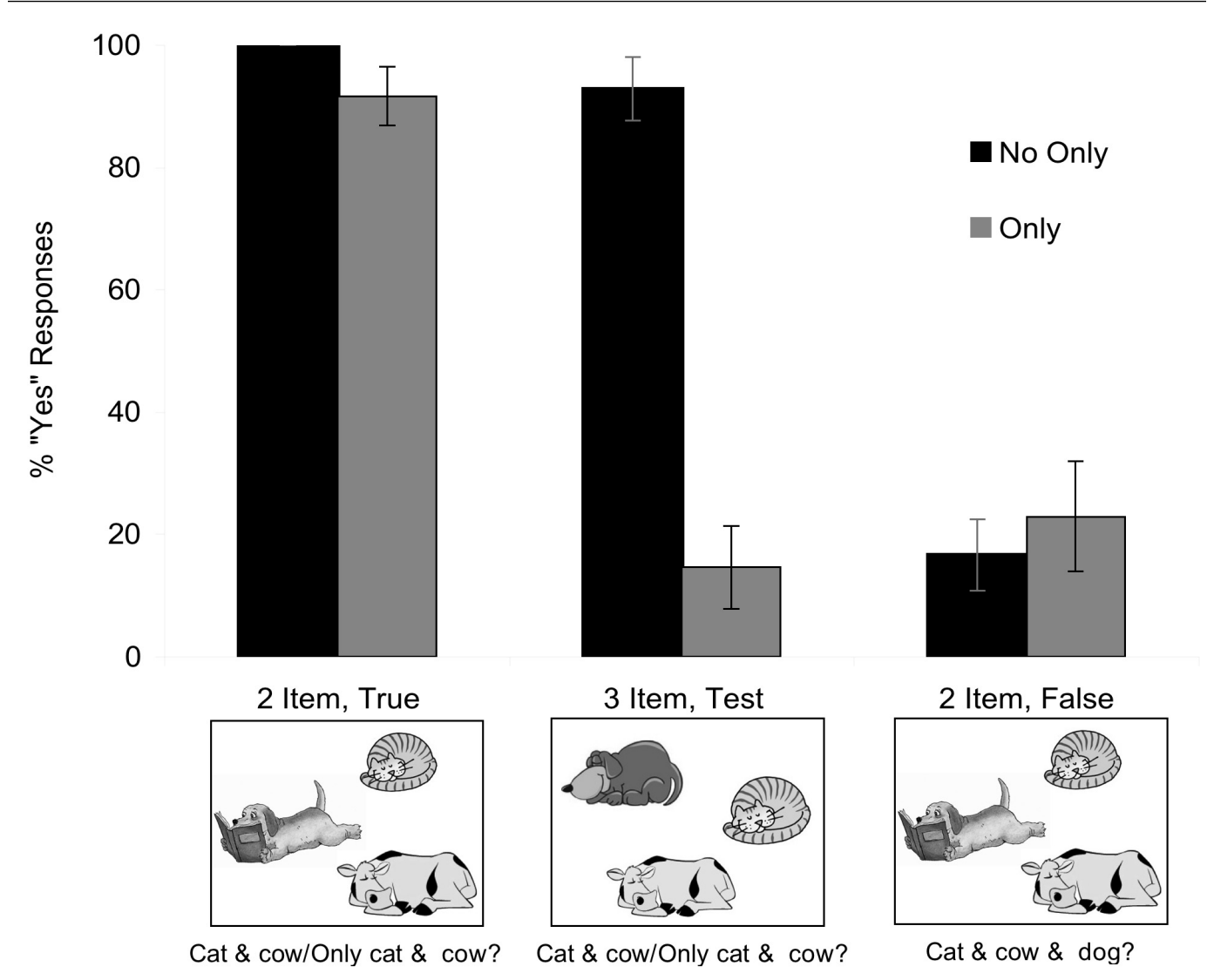

Figure 3 Percentage of children who said yes to questions in the Contextual alternatives conditions. Error bars represent standard error. 
utterances containing the word some.

\section{General Discussion and Conclusion}

The results from this study are consistent with the hypothesis that children's knowledge of scalar alternatives places a significant constraint on their ability to compute strengthened meanings. In the experiment, children were able to assign strengthened interpretations to utterances when they included the focus element only, as long as alternatives were provided contextually. For context-independent scales - e.g., somelall - children failed to compute a strengthened meaning most of the time, even when only was added. Since only forces strengthening grammatically (and clearly did so for contextual alternatives), no pragmatic inference was required. Thus, children's failure to derive strengthened readings for some is consistent with a hypothesized failure to generate relevant scalar alternatives - in this case the quantifier all.

Besides showing that children have difficulty generating relevant alternatives for scales like $\langle$ some, all $\rangle$, these data also suggest that, contrary to speculation in the literature (e.g., Chierchia et al. 2001; Pouscoulous et al. 2007; Reinhart 2004), children's difficulties are not straightforwardly attributable to limitations of working memory, task demand, or other types of processing constraints. In our study, we found that children were perfectly capable of deriving strengthened interpretations for utterances that involved contextual alternatives, but failed for identical sentences that involved some. This is despite the fact that similar processes were involved across conditions. In both cases, children were required to (1) represent the basic meaning of the utterance, (2) generate relevant alternatives, (3) restrict alternatives to those that are stronger (i.e., more informative), and (4) negate the stronger alternatives to strengthen the utterance. Our suggestion is that whereas a clear difference between conditions is found for Step 2, there is no clear way that differences between conditions at other steps in the process could predict children's behaviors. This is because when children are freed from the need to generate alternatives - i.e., they are specified contextually - they succeed without difficulty.

Could a processing limit explain a failure to access alternatives? As noted in the introduction, Chierchia et al. (2001) speculate that children's failure to compute implicatures for or vs. and is due to a failure to access alternatives, and that this failure is related to processing limits. Although Chierchia et al. do not describe a specific mechanism in their account, there are several possibilities. One solution, consistent with our account, is that if scalar items are not associated with one another as scale mates, then children may need to expend additional resources to construct scales in an ad hoc fashion, and fail in their effort to do so on most occasions. This might explain why children become more likely to compute implicatures when they 
Quantity implicature and access to scalar alternatives

are first trained to reject descriptions that are underinformative (e.g., the use of animal to describe a dog). By encouraging children to actively search alternatives which they might otherwise avoid due to the additional effort required - such training might compensate for weak knowledge of scales, allowing children to succeed during the course of the experiment. Consistent with this, training effects are transient, and do not persist after a delay of one week (Guasti et al. 2005). By this account, if quantifiers were associated as members of scales (not a processing cost in itself) children would access alternatives without problem, as they do for numerals. A second possibility, which we consider less plausible, is that children have difficulty holding scalar alternatives in working memory as they compute inferences, thereby explaining why quantifiers would be harder than contextual alternatives (which remain visible to children as they process the sentence). The problem here is that such an account cannot explain children's relative success with other scales. When scale members are strongly associated via memorization, as with numerals, children have no difficulty holding alternatives in working memory and computing inferences.

From a broader perspective, the idea that children's difficulties are scale-specific, rather than due to pragmatic immaturity, is also consistent with reports of pragmatic sophistication in other domains, such as noun learning (see Baldwin 1993; Clark 1987, 1988; Markman 1989; Tomasello 1992). For example, children assume that words have mutually exclusive meanings from very early in language learning (Clark 1987, 1988; Markman 1989; Carey \& Bartlett 1978). Children do not apply mutual exclusivity if they believe a novel word is not at the same level of description as the label for the known object, or if they are told the word is from another language (Au \& Glusman 1990). In these cases, the known label is not considered a relevant alternative to the novel label. These simple inferences, though distinct from implicatures in many ways, nonetheless require both pragmatic understanding (including ascription of speaker intent), and the processing abilities needed to entertain and restrict possible alternatives. These abilities would be difficult to explain if children's difficulties with scalar implicature were due to processing limits or a general insensitivity to conversational pragmatics.

What must children learn about scales to use them for implicature and other types of strengthening? One possibility is that, in absence of brute memorization as with numerals, children may group lexical items as scale mates by a gradual association of syntactically replaceable alternatives (cf. Katzir 2007). Alternatively, scale members may be associated as children hear them explicitly contrasted in conversation - e.g., Give me some of the cake, but not all of it. In each case, some form of gradual learning would be required. Currently, no empirical studies address how scales might be constructed, and even in adults the psychological status of scales is poorly understood - i.e., which words and phrases form scales and which do not. Future studies should explore the nature of scales both in adults and children, 
to better understand how scales are constructed in acquisition to support pragmatic inference.

\section{References}

Au, Terry K. \& Marianna Glusman. 1990. The principle of mutual exclusivity in word learning: To honor or not to honor? Child Development 61(5). 1474-1490. doi: $10.2307 / 1130757$.

Baldwin, Dare A. 1993. Infants' ability to consult the speaker for clues to word reference. Journal of Child Language 20(2). 395-418. doi:10.1017/S0305000900008345.

Barner, David \& Asaf Bachrach. 2010. Inference and exact numerical representation in early language development. Cognitive Psychology 60(1). 40-62. doi:10.1016/j.cogpsych.2009.06.002.

Barner, David, Katherine Chow \& Shu-Ju Yang. 2009. Finding one's meaning: A test of the relation between quantifiers and integers in language development. Cognitive Psychology 58(2). 195-219. doi:10.1016/j.cogpsych.2008.07.001.

Bloom, Paul. 2000. How children learn the meanings of words. Cambridge, MA: MIT Press.

Breheny, Richard. 2008. A new look at the semantics and pragmatics of numerically quantified noun phrases. Journal of Semantics 25(2). 93-140. doi:10.1093/jos/ffm016.

Carey, Susan. 2009. The origin of concepts. New York: Oxford University Press.

Carey, Susan \& Elsa Bartlett. 1978. Acquiring a single new word. Proceedings of the Stanford Child Language Conference 15. 17-29.

Chierchia, Gennaro, Stephen Crain, Maria T. Guasti, Andrea Gualmini \& Luisa Meroni. 2001. The acquisition of disjunction: Evidence for a grammatical view of scalar implicatures. In Proceedings of the 25th Boston University Conference on Language Development, Somerville, MA: Cascadilla Press.

Clark, Eve. 1987. The principle of contrast: A constraint on language acquisition. In B. MacWhinney (ed.), Mechanisms of language acquisition, 1-33.

Clark, Eve. 1988. On the logic of contrast. Journal of Child Language 15(2). 317-335. doi:10.1017/S0305000900012393.

Condry, Kirsten F. \& Elizabeth S. Spelke. 2008. The development of language and abstract concepts: The case of natural number. Journal of Experimental Psychology: General 137(1). 22-38. doi:10.1037/0096-3445.137.1.22.

Crain, Stephen, Takuya Goro \& Utako Minai. 2007. Hidden units in child language. In A.C. Schalley \& D. Khlentzos (eds.), Mental states. volume 1: Evolution function, nature, 249-277. Amsterdam: John Benjamins. 
Quantity implicature and access to scalar alternatives

Fuson, Karen C. 1988. Children's counting and concepts of number. New York: Springer-Verlage.

Gathercle, Susan E. \& Alan D. Baddeley. 1990. Phonological memory deficits in language disordered children: Is there a causal connection? Journal of Memory and Language 29(3). 336-360. doi:10.1016/0749-596X(90)90004-J.

Goro, Takuya, Utako Minai \& Stephen Crain. 2005. Two disjunctions for the price of only one. In Proceedings of the 29th Boston University Conference on Language Development, Somerville, MA: Cascadilla Press.

Goro, Takuya, Utako Minai \& Stephen Crain. 2006. Bringing out the logic in child language. In Proceedings of the 35th Annual Meeting of the North East Linguistic Society, Amherst, MA: GLSA Publications.

Grice, Herbert Paul. 1978. Further notes on logic and conversation. In P. Cole (ed.), Syntax and semantics: Vol. 9. pragmatics, 113-128. New York: Academic Press.

Grice, Herbert Paul. 1989. Studies in the way of words. Cambridge, MA: Harvard University Press.

Gualmini, Andrea, Stephen Crain, Luisa Meroni, Gennaro Chierchia \& Maria T. Guasti. 2001. At the semantics/pragmatics interface in child language. In Proceedings of Semantics and Linguistic Theory XI, Cornell, Ithaca: CLC Publications.

Guasti, Maria T., Gennaro Chierchia, Stephen Crain, Francesca Foppolo, Andrea Gualmini \& Luisa Meroni. 2005. Why children and adults sometimes (but not always) compute implicatures. Language and Cognitive Processes 20(5). 667-696. doi:10.1080/01690960444000250.

Hirschberg, Julia. 1985. A theory of scalar implicature. Philadelphia, PA: University of Pennsylvania dissertation.

Horn, Laurence. 1972. On the semantic properties of the logical operators in English. Los Angeles, CA: UCLA dissertation.

Horn, Laurence. 1989. A natural history of negation. Chicago: University of Chicago Press.

Huang, Yi Ting \& Jesse Snedeker. 2009a. Online interpretation of scalar quantifiers: Insight into the semantics-pragmatics interface. Cognitive Psychology 58(3). 376-415. doi:10.1016/j.cogpsych.2008.09.001.

Huang, Yi Ting \& Jesse Snedeker. 2009b. Semantic meaning and pragmatic interpretation in five-year-olds: Evidence from real time spoken language comprehension. Developmental Psychology 45(6). 1723-1739. doi:10.1037/a0016704.

Huang, Yi Ting, Jesse Snedeker \& Elizabeth Spelke. under review. What exactly do numbers mean?

Hurewitz, Felicia, Anna Papafragou, Lila Gleitman \& Rochel Gelman. 2006. Asymmetries in the acquisition of numbers and quantifiers. Language Learning and Development 2(2). 77-96. doi:10.1207/s154733411ld0202_1. 
Katzir, Roni. 2007. Structurally-defined alternatives. Linguistics and Philosophy 30(6). 669-690. doi:10.1007/s10988-008-9029-y.

Markman, Ellen M. 1989. Categorization and naming in children. Cambridge, MA: MIT Press.

Matsumoto, Yo. 1995. The conversational condition on Horn scales. Linguistics and Philosophy 18(1). 21-60. doi:10.1007/BF00984960.

Musolino, Julien. 2004. The semantics and acquisition of number words: Integrating linguistic and developmental perspectives. Cognition 93(1). 1-41. doi:10.1016/j.cognition.2003.10.002.

Musolino, Julien. 2006. On the semantics of the subset principle. Language Learning and Development 2(3). 195-218. doi:10.1207/s154733411ld0203_3.

Musolino, Julien \& Jeff Lidz. 2006. Why children aren't universally successful with quantification. Linguistics 44(4). 817-852. doi:10.1515/LING.2006.026.

Noveck, Ira \& Andres Posada. 2003. Characterizing the time course of an implicature: An evoked potentials study. Brain and Language 85(2). 203-210. doi:10.1016/S0093-934X(03)00053-1.

Noveck, Ira A. 2001. When children are more logical than adults: Experimental investigation of scalar implicatures. Cognition 78(2). 165-188. doi:10.1016/S0010-0277(00)00114-1.

Papafragou, Anna. 2006. From scalar semantics to implicature: Children's interpretation of aspectuals. Journal of Child Language 33(4). 721-757. doi:10.1017/S0305000906007550.

Papafragou, Anna \& Julie Musolino. 2003. Scalar implicatures: Experiments at the semantics-pragmatics interface. Cognition 86(3). 253-282. doi:10.1016/S00100277(02)00179-8.

Papafragou, Anna \& Niki Tantalou. 2004. Children's computation of implicatures. Language Acquisition 12(1). 71-82. doi:10.1207/s15327817la1201_3.

Pouscoulous, Nausicaa, Ira A. Noveck, Guy Politzer \& Anne Bastide. 2007. A developmental investigation of processing costs in implicature production. Language Acquisition 14(4). 347-375. doi:10.1080/10489220701600457.

Reinhart, Tanya. 2004. The processing cost of reference-set computation: Acquisition of stress shift and focus. Language Acquisition 12. 109-155.

Repacholi, Betty M. \& Alison Gopnik. 1997. Early reasoning about desires: Evidence from 14- and 18-month-olds. Developmental Psychology 33(1). 12 - 21. doi:10.1037/0012-1649.33.1.12.

Rips, Lance. 1975. Quantification and semantic memory. Cognitive Psychology 7(3). 307-340. doi:10.1016/0010-0285(75)90014-6.

Smith, Carol L. 1980. Quantifiers and question answering in young children. Journal of Experimental Child Psychology 30(2). 191-205. doi:10.1016/00220965(80)90057-0. 
Quantity implicature and access to scalar alternatives

Tomasello, Michael. 1992. The social bases of language acquisition. Social Development 1(1). 67-87. doi:10.1111/j.1467-9507.1992.tb00135.x.

Wynn, Karen. 1992. Children's acquisition of number words and the counting system. Cognitive Psychology 24(2). 220-251. doi:10.1016/0010-0285(92)90008-P.

David Barner

Department of Psychology

University of California, San Diego

9500 Gilman Drive \#0109

La Jolla, CA 92093-0109

barner@ucsd.edu

Alan Bale

Linguistics Program, Concordia University

1455 de Maisonneuve W., Montréal, Québec

Canada H3G 1M8

alancbale@gmail.com
Neon Brooks

Department of Psychology

The University of Chicago

5848 South University Avenue

Chicago, IL 60637

neonblue@uchicago.edu 